\title{
On a canonical nine-body problem. Integrable Ermakov decomposition via reciprocal transformations
}

Colin Rogers

To cite this article: Colin Rogers (2019) On a canonical nine-body problem. Integrable Ermakov decomposition via reciprocal transformations, Journal of Nonlinear Mathematical Physics 26:1, 98-106, DOI:

https://doi.org/10.1080/14029251.2019.1544792

To link to this article: https://doi.org/10.1080/14029251.2019.1544792

Published online: 04 January 2021 


\title{
On a canonical nine-body problem. Integrable Ermakov decomposition via reciprocal transformations
}

\author{
Colin Rogers \\ School of Mathematics and Statistics, \\ The University of New South Wales, \\ Sydney, NSW2052, Australia \\ c.rogers@unsw.edu.au
}

Received 16 July 2018

Accepted 12 August 2018

\begin{abstract}
Here, a recently introduced nine-body problem is shown to be decomposable via a novel class of reciprocal transformations into a set of integrable Ermakov systems. This Ermakov decomposition is exploited to construct more general integrable nine-body systems in which the canonical nine-body system is embedded.
\end{abstract}

Keywords: Many Body; Ermakov; Reciprocal.

2000 Mathematics Subject Classification: MSC 37K10

\section{Introduction}

The study of many-body problems with their established importance in both classical and quantum mechanics has an extensive literature motivated, in particular, by the pioneering models of Calogero $[4,5]$, Moser [20] and Sutherland [48,49]. The surveys [6,19,22] and the literature cited therein may be consulted in this regard.

In recent work in [25], non-autonomous extensions of 3-body and 4-body systems incorporating those set down in $[2,7,17]$ have been shown to be decomposible into integrable multi-component Ermakov systems. Two-component Ermakov systems adopt the form [23, 24,26]

$$
\begin{aligned}
& \ddot{\alpha}+\omega(t) \alpha=\frac{1}{\alpha^{2} \beta} \Phi(\beta / \alpha), \\
& \ddot{\beta}+\omega(t) \beta=\frac{1}{\alpha \beta^{2}} \Psi(\alpha / \beta)
\end{aligned}
$$

and admit a distinctive integral of motion, namely, the invariant

$$
\mathscr{E}=\frac{1}{2}(\alpha \dot{\beta}-\beta \dot{\alpha})^{2}+\int^{\beta / \alpha} \Phi(z) d z+\int^{\alpha / \beta} \Psi(w) d w
$$

together with concomitant nonlinear superposition principles. Here, a dot indicates a derivative with respect to the independent variable $t$. Ermakov systems of the type (1.1) have diverse physical applications, notably in nonlinear optics $[8,13-16,27,28,51]$. There, in particular, they arise in the description of the evolution of size and shape of the light spot and wave front in elliptical Gaussian beams. In 2+1-dimensional rotating shallow water hydrodynamics, Hamiltonian two-component Ermakov systems of the type (1.1) have been derived in [29] which describe the time evolution of the 
semi-axes of the elliptic moving shoreline on an underlying circular paraboloidal basin. ErmakovRay-Reid systems have also been obtained in magnetogasdynamics in $[30,50]$ and novel pulsrodontype phenomena thereby isolated analogous to that observed in an elliptic warm-core oceanographic eddy context [31,47]. Nonlinear coupled systems of Ermakov-type have also been shown in [32] to arise in the description of gas cloud evolution as originally investigated by Dyson [11]. In [28], it was shown that the occurrence of integrable Hamiltonian Ermakov-Ray-Reid systems in nonlinear physics and continuum mechanics, remarkably, extends to the spiralling elliptic soliton system of [9] and to its generalisation in the Bose-Einstein context of [1].

In connection with many-body problems, a nine-body system has recently been investigated in detail in [3]. The mode of treatment for this canonical system was thereby extended to a class of $3^{k}$ many-body problems. Here, an alternative approach to nine-body problems of the type in [3] is adopted wherein they are shown via a novel class of reciprocal transformations to be decomposible into an equivalent set of integrable Ermakov systems. This Ermakov connection is exploited here to embed the original nine-body system in a wide solvable class involving a triad of arbitrary functions $J_{1}(y / x), J_{2}(y / x)$ and $J_{3}(y / x)$ where $x, y$ are Jacobi variables. These $J_{i}(y / x), i=1,2,3$ are associated with a general parametrisation of two-component Hamiltonian Ermakov systems as originally introduced in [29].

\section{A Class of Nine-Body Problems. Ermakov Reduction}

Here, we consider a class of nine-body problems

$$
\ddot{x}_{i}=\frac{\partial Z}{\partial x_{i}}, \quad i=1,2, \ldots, 9
$$

of the type introduced in [3] with

$$
\begin{aligned}
Z= & \sum_{1 \leqslant i \leqslant j<3} \frac{\lambda_{1,1}}{\left(x_{i}-x_{j}\right)^{2}}+\sum_{4 \leqslant i<j \leqslant 6} \frac{\lambda_{2,1}}{\left(x_{i}-x_{j}\right)^{2}}+\sum_{7 \leqslant i<j \leqslant 9} \frac{\lambda_{3,1}}{\left(x_{i}-x_{j}\right)^{2}} \\
& +\sum_{1 \leqslant i<j \leqslant 3} \frac{3 \lambda_{1,2}}{\left(x_{3 i-2}+x_{3 i-1}+x_{3 i}-x_{3 j-2}-x_{3 j-1}-x_{3 j}\right)^{2}}-\frac{\delta}{2\left(\sum_{i=1}^{9} x_{i}\right)^{2}}+\frac{\mu}{\sum_{i=1}^{9} x_{i}^{2}} .
\end{aligned}
$$

It is noted that $\delta=0$ in the system investigated in [3].

Jacobi and centre of mass co-ordinates are now introduced according to

$$
\begin{gathered}
x=\frac{1}{\sqrt{2}}\left(x_{1}-x_{2}\right), \quad y=\frac{1}{\sqrt{6}}\left(x_{1}+x_{2}-2 x_{3}\right), \\
z=\frac{1}{\sqrt{2}}\left(x_{4}-x_{5}\right), \quad v=\frac{1}{\sqrt{6}}\left(x_{4}+x_{5}-2 x_{6}\right), \\
r=\frac{1}{\sqrt{2}}\left(x_{7}-x_{8}\right), \quad s=\frac{1}{\sqrt{6}}\left(x_{7}+x_{8}-2 x_{9}\right) \\
w=\frac{1}{\sqrt{3}}\left(x_{1}+x_{2}+x_{3}\right), p=\frac{1}{\sqrt{3}}\left(x_{4}+x_{5}+x_{6}\right), q=\frac{1}{\sqrt{3}}\left(x_{7}+x_{8}+x_{9}\right) .
\end{gathered}
$$

Under this linear transformation, the invariance property

$$
\Sigma:=x_{1}^{2}+x_{2}^{2}+\cdots+x_{9}^{2}=x^{2}+y^{2}+z^{2}+v^{2}+r^{2}+s^{2}+w^{2}+p^{2}+q^{2}
$$


is seen to hold. Moreover,

$$
\begin{array}{lll}
x_{1}=\frac{1}{\sqrt{2}} x+\frac{1}{\sqrt{6}} y+\frac{1}{\sqrt{3}} w, & x_{2}=-\frac{1}{\sqrt{2}} x+\frac{1}{\sqrt{6}} y+\frac{1}{\sqrt{3}} w, & x_{3}=-\sqrt{\frac{2}{3}} y+\frac{1}{\sqrt{3}} w, \\
x_{4}=\frac{1}{\sqrt{2}} z+\frac{1}{\sqrt{6}} v+\frac{1}{\sqrt{3}} p, & x_{5}=-\frac{1}{\sqrt{2}} z+\frac{1}{\sqrt{6}} v+\frac{1}{\sqrt{3}} p, & x_{6}=-\sqrt{\frac{2}{3}} v+\frac{1}{\sqrt{3}} p, \\
x_{7}=\frac{1}{\sqrt{2}} r+\frac{1}{\sqrt{6}} s+\frac{1}{\sqrt{3}} q, & x_{8}=-\frac{1}{\sqrt{2}} r+\frac{1}{\sqrt{6}} s+\frac{1}{\sqrt{3}} q, & x_{9}=-\sqrt{\frac{2}{3}} s+\frac{1}{\sqrt{3}} q .
\end{array}
$$

'In extenso' on re-labelling, (2.2) yields

$$
\begin{aligned}
Z= & \frac{\lambda_{1}}{\left(x_{1}-x_{2}\right)^{2}}+\frac{\lambda_{2}}{\left(x_{2}-x_{3}\right)^{2}}+\frac{\lambda_{3}}{\left(x_{3}-x_{1}\right)^{2}} \\
& +\frac{\mu_{1}}{\left(x_{4}-x_{5}\right)^{2}}+\frac{\mu_{2}}{\left(x_{5}-x_{6}\right)^{2}}+\frac{\mu_{3}}{\left(x_{6}-x_{4}\right)^{2}} \\
& +\frac{v_{1}}{\left(x_{7}-x_{8}\right)^{2}}+\frac{v_{2}}{\left(x_{8}-x_{9}\right)^{2}}+\frac{v_{3}}{\left(x_{9}-x_{7}\right)^{2}} \\
& +\frac{\sigma_{1}}{\left(x_{1}+x_{2}+x_{3}-x_{4}-x_{5}-x_{6}\right)^{2}}+\frac{\sigma_{2}}{\left(x_{4}+x_{5}+x_{6}-x_{7}-x_{8}-x_{9}\right)^{2}} \\
& +\frac{\sigma_{3}}{\left(x_{7}+x_{8}+x_{9}-x_{1}-x_{2}-x_{3}\right)^{2}} \\
& -\frac{\delta}{2\left(x_{1}+x_{2}+\ldots+x_{9}\right)^{2}}+\frac{\mu}{x_{1}^{2}+x_{2}^{2}+\ldots+x_{9}^{2}} .
\end{aligned}
$$

In terms of the Jacobi and centre of mass co-ordinates, the 9-body system determined by (2.1) with $Z$ given by (2.6) becomes

$$
\begin{aligned}
& \ddot{x}+\frac{2 \mu x}{\Sigma^{2}}=-\frac{\lambda_{1}}{x^{3}}+\frac{4 \lambda_{2}}{(\sqrt{3} y-x)^{3}}-\frac{4 \lambda_{3}}{(x+\sqrt{3} y)^{3}}, \\
& \ddot{y}+\frac{2 \mu y}{\Sigma^{2}}=-\frac{4 \sqrt{3} \lambda_{2}}{(\sqrt{3} y-x)^{3}}-\frac{4 \sqrt{3} \lambda_{3}}{(x+\sqrt{3} y)^{3}}, \\
& \ddot{z}+\frac{2 \mu z}{\Sigma^{2}}=-\frac{\mu_{1}}{z^{3}}+\frac{4 \mu_{2}}{(\sqrt{3} v-z)^{3}}-\frac{4 \mu_{3}}{(z+\sqrt{3} v)^{3}}, \\
& \ddot{v}+\frac{2 \mu v}{\Sigma^{2}}=-\frac{4 \sqrt{3} \mu_{2}}{(\sqrt{3} v-z)^{3}}-\frac{4 \sqrt{3} \mu_{3}}{(z+\sqrt{3} v)^{3}}, \\
& \ddot{r}+\frac{2 \mu r}{\Sigma^{2}}=-\frac{v_{1}}{r^{3}}+\frac{4 v_{2}}{(\sqrt{3} s-r)^{3}}-\frac{4 v_{3}}{(r+\sqrt{3} s)^{3}}, \\
& \ddot{s}+\frac{2 \mu s}{\Sigma^{2}}=-\frac{4 \sqrt{3} v_{2}}{(\sqrt{3} s-r)^{3}}-\frac{4 \sqrt{3} v_{3}}{(r+\sqrt{3} s)^{3}}, \\
& \ddot{w}+\frac{2 \mu w}{\Sigma^{2}}=\frac{2}{3}\left[-\frac{\sigma_{1}}{(w-p)^{3}}+\frac{\sigma_{3}}{(q-w)^{3}}\right]+\frac{\delta}{3(w+p+q)^{3}}, \\
& \ddot{p}+\frac{2 \mu p}{\Sigma^{2}}=\frac{2}{3}\left[\frac{\sigma_{1}}{(w-p)^{3}}-\frac{\sigma_{2}}{(p-q)^{3}}\right]+\frac{\delta}{3(w+p+q)^{3}}, \\
& \ddot{q}+\frac{2 \mu q}{\Sigma^{2}}=\frac{2}{3}\left[\frac{\sigma_{2}}{(p-q)^{3}}-\frac{\sigma_{3}}{(q-w)^{3}}\right]+\frac{\delta}{3(w+p+q)^{3}} .
\end{aligned}
$$




\section{Application of a Reciprocal Transformation}

Reciprocal-type transformations have diverse physical applications in such areas as gasdynamics and magnetogasdynamics [33,34], the solution of nonlinear moving boundary problems [12,35-38], the analysis of oil/water migration through a porous medium [39] and in Cattaneo-type hyperbolic nonlinear heat conduction [40]. Reciprocal transformations have also been applied in the theory of discontinuity-wave propagation [10]. In soliton theory, the conjugation of reciprocal and gauge transformations has been used to link integrable equations and the inverse scattering schemes in which they are embedded [18,21,41-45]. Here, a novel class of reciprocal transformations is introduced in the present context of many-body theory. This is used to reduce the nine-body system (2.7) in Jacobi and centre of mass co-ordinates to an equivalent set of integrable two-component Ermakov systems of the type (1.1) augmented by a classical single component Ermakov equation in a centre of mass component.

Thus, the class of reciprocal transformations

$$
\left.\begin{array}{c}
x^{*}=x / \rho, \quad y^{*}=y / \rho, \quad z^{*}=z / \rho, \\
v^{*}=v / \rho, \quad s^{*}=s / \rho, \quad w^{*}=w / \rho, \quad p^{*}=p / \rho, \quad q^{*}=q / \rho \\
\rho^{*}=\rho^{-1}, \quad d t^{*}=\rho^{-2} d t
\end{array}\right\} \quad \mathbb{R}^{*}
$$

such that $\mathbb{R}^{* 2}=\mathrm{I}$ is introduced wherein $\rho$ is governed by the base equation

$$
\ddot{\rho}+2 \mu \rho \Sigma^{-2}=0
$$

under $\mathbb{R}^{*}$, the nine-body system becomes

$$
\begin{aligned}
& x_{t^{*} t^{*}}^{*}=-\frac{\lambda_{1}}{x^{* 3}}+\frac{4 \lambda_{2}}{\left(\sqrt{3} y^{*}-x^{*}\right)^{3}}-\frac{4 \lambda_{3}}{\left(x^{*}+\sqrt{3} y^{*}\right)^{3}}, \\
& y_{t^{*} t^{*}}^{*}=-\frac{4 \sqrt{3} \lambda_{2}}{\left(\sqrt{3} y^{*}-x^{*}\right)^{3}}-\frac{4 \sqrt{3} \lambda_{3}}{\left(x^{*}+\sqrt{3} y^{*}\right)^{3}}, \\
& z_{t^{*} t^{*}}^{*}=-\frac{\mu_{1}}{z^{* 3}}+\frac{4 \mu_{2}}{\left(\sqrt{3} v^{*}-z^{*}\right)^{3}}-\frac{4 \mu_{3}}{\left(z^{*}+\sqrt{3} v^{*}\right)^{3}}, \\
& v_{t^{*} t^{*}}^{*}=-\frac{4 \sqrt{3} \mu_{2}}{\left(\sqrt{3} v^{*}-z^{*}\right)^{3}}-\frac{4 \sqrt{3} \mu_{3}}{\left(z^{*}+\sqrt{3} v^{*}\right)^{3}}, \\
& r_{t^{*} t^{*}}^{*}=-\frac{v_{1}}{r^{* 3}}+\frac{4 v_{2}}{\left(\sqrt{3} s^{*}-r^{*}\right)^{3}}-\frac{4 v_{3}}{\left(r^{*}+\sqrt{3} s^{*}\right)^{3}}, \\
& s_{t^{*} t^{*}}^{*}=-\frac{4 \sqrt{3} v_{2}}{\left(\sqrt{3} s^{*}-r^{*}\right)^{3}}-\frac{4 \sqrt{3} v_{3}}{\left(r^{*}+\sqrt{3} s^{*}\right)^{3}},
\end{aligned}
$$

together with

$$
\begin{aligned}
& w_{t^{*} t^{*}}^{*}=\frac{2}{3}\left[-\frac{\sigma_{1}}{\left(w^{*}-p^{*}\right)^{3}}+\frac{\sigma_{3}}{\left(q^{*}-w^{*}\right)^{3}}\right]+\frac{\delta}{3 R^{* 3}}, \\
& p_{t^{*} t^{*}}^{*}=\frac{2}{3}\left[\frac{\sigma_{1}}{\left(w^{*}-p^{*}\right)^{3}}-\frac{\sigma_{2}}{\left(p^{*}-q^{*}\right)^{3}}\right]+\frac{\delta}{R^{* 3}}, \\
& q_{t^{*} t^{*}}^{*}=\frac{2}{3}\left[\frac{\sigma_{2}}{\left(p^{*}-q^{*}\right)^{3}}-\frac{\sigma_{3}}{\left(q^{*}-w^{*}\right)^{3}}\right]+\frac{\delta}{R^{* 3}},
\end{aligned}
$$


where

$$
R^{*}=\frac{R}{\rho}, \quad R=\frac{1}{\sqrt{3}}\left(x_{1}+x_{2}+\cdots+x_{9}\right)=w+p+q .
$$

In the above, (3.3) constitute three copies of integrable Hamiltonian Ermakov systems of the same kind as obtained in [25] for the original 3-body system of Calogero [4].

On introduction of the translational change of variables

$$
W^{*}=w^{*}-p^{*}, \quad P^{*}=p^{*}-q^{*}
$$

the system (3.4) produces the two-component Ermakov system

$$
\begin{aligned}
W_{t^{*} t^{*}}^{*} & =\frac{2}{3}\left[-\frac{2 \sigma_{1}}{W^{* 3}}+\frac{\sigma_{2}}{P^{* 3}}-\frac{\sigma_{3}}{\left(W^{*}+P^{*}\right)^{3}}\right], \\
P_{t^{*} t^{*}}^{*} & =\frac{2}{3}\left[\frac{\sigma_{1}}{W^{* 3}}-\frac{2 \sigma_{2}}{P^{* 3}}-\frac{\sigma_{3}}{\left(W^{*}+P^{*}\right)^{3}}\right]
\end{aligned}
$$

while addition of the constituent members of (3.3)-(3.4) shows that

$$
R_{t^{*} t^{*}}^{*}=\frac{\delta}{3 R^{* 3}}
$$

namely, a single component Ermakov equation in $R^{*}$.

The triad (3.3) constitutes three de-coupled Ermakov systems of the type (1.1) and with associated Hamiltonians

$$
\begin{aligned}
& \mathscr{H}_{\mathrm{I}}=\frac{1}{2}\left[x_{t^{*}}^{* 2}+y_{t^{*}}^{* 2}\right]+\frac{1}{x^{* 2}}\left[\frac{\lambda_{1}}{2}+\frac{2 \lambda_{2}}{\left(\sqrt{3} y^{*} / x^{*}-1\right)^{3}}+\frac{2 \lambda_{3}}{\left(\sqrt{3} y^{*} / x^{*}+1\right)^{3}}\right], \\
& \mathscr{H}_{\mathrm{II}}=\frac{1}{2}\left[z_{t^{*}}^{* 2}+v_{t^{*}}^{* 2}\right]+\frac{1}{z^{* 2}}\left[\frac{\mu_{1}}{2}+\frac{2 \mu_{2}}{\left(\sqrt{3} v^{*} / z^{*}-1\right)^{3}}+\frac{2 \mu_{3}}{\left(\sqrt{3} v^{*} / z^{*}+1\right)^{3}}\right], \\
& \mathscr{H}_{\mathrm{III}}=\frac{1}{2}\left[r_{t^{*}}^{* 2}+s_{t^{*}}^{* 2}\right]+\frac{1}{r^{* 2}}\left[\frac{v_{1}}{2}+\frac{2 v_{2}}{\left(\sqrt{3} s^{*} / r^{*}-1\right)^{3}}+\frac{2 v_{3}}{\left(\sqrt{3} s^{*} / r^{*}+1\right)^{3}}\right] .
\end{aligned}
$$

The sub-system (3.4), on the other hand admits the Hamiltonian

$$
\begin{aligned}
\mathscr{H}_{\mathrm{IV}}=\frac{1}{2}\left[w_{t^{*}}^{* 2}+p_{t^{*}}^{* 2}+q_{t^{*}}^{* 2}\right]-\frac{1}{3}\left[\frac{\sigma_{1}}{\left(w^{*}-p^{*}\right)^{2}}+\frac{\sigma_{2}}{\left(p^{*}-q^{*}\right)^{2}}+\frac{\sigma_{3}}{\left(w^{*}-q^{*}\right)^{2}}\right] \\
+\frac{\delta}{6\left(w^{*}+p^{*}+q^{*}\right)^{2}}
\end{aligned}
$$

that is,

$$
\mathscr{H}_{\mathrm{IV}}=\frac{1}{6}\left[2 W_{t^{*}}^{* 2}+\left(P_{t^{*}}^{*}+R_{t^{*}}^{*}\right)^{2}\right]-\frac{1}{3}\left[\frac{\sigma_{1}}{W^{* 2}}+\frac{\sigma_{2}}{P^{* 2}}+\frac{\sigma_{3}}{\left(W^{*}+P^{*}\right)^{2}}\right]+\frac{\delta}{6 R^{* 2}}
$$

wherein $R^{*}$ is determined by the Ermakov equation (3.8).

Thus, the nine-body system encapsulated in (3.3)-(3.4) is seen to be reducible to a quartet of two-component Ermakov systems of the classical type (1.1), augmented by the single component Ermakov equation (3.8) in $R^{*}$. Moreover, the Ermakov-Ray-Reid systems in (3.3) and (3.7), in addition to their admittance of characteristic Ermakov invariants, also admit second integrals of motion, namely, the Hamiltonians $\mathscr{H}_{\mathrm{I}} \ldots \mathscr{H}_{\mathrm{IV}}$ and hence are integrable. 
The preceding determines $x^{*}\left(t^{*}\right), y^{*}\left(t^{*}\right), z^{*}\left(t^{*}\right), v^{*}\left(t^{*}\right), r^{*}\left(t^{*}\right), s^{*}\left(t^{*}\right)$ together with $w^{*}-$ $p^{*}, p^{*}-q^{*}$ and $R^{*}=w^{*}+p^{*}+q^{*}$ and hence $w^{*}\left(t^{*}\right), p^{*}\left(t^{*}\right)$ and $q^{*}\left(t^{*}\right)$. In addition, the base equation (3.2) in $\rho$, in the reciprocal variables becomes

$$
\rho_{t^{*} t^{*}}^{*}-\frac{2 \mu \rho^{*}}{\left[x^{*}+y^{* 2}+\cdots+q^{* 2}\right]^{2}}=0
$$

The latter becomes determinate on insertion of $x^{*}, y^{*}, \ldots, q^{*}$ as obtained by means of the integrable Hamiltonian Ermakov systems. With $\rho^{*}\left(t^{*}\right)$ to hand, $t=t\left(t^{*}\right)$ is determined by the pair of reciprocal relations

$$
\rho^{*}=\rho^{-1}, \quad d t^{*}=\rho^{-2} d t
$$

so that

$$
d t=\rho^{*-2} d t^{*}
$$

while the original Jacobi and centre of mass variables $x(t), y(t), \ldots, q(t)$ are given parametrically via $t^{*}$ by the residual reciprocal relations

$$
x=x^{*}\left(t^{*}\right) / \rho^{*}\left(t^{*}\right), \quad y=y^{*}\left(t^{*}\right) / \rho^{*}\left(t^{*}\right), \quad \ldots, \quad q=q^{*}\left(t^{*}\right) / \rho^{*}\left(t^{*}\right) .
$$

\section{An Extended Solvable Nine-Body System. Application of the Ermakov Connection}

The isolation of integrable nonlinear systems of Ermakov-type is a subject of current interest (see e.g. [46]). Here, the Ermakov reduction of the nine-body system (2.1)-(2.2) may be exploited to embed it in a more general novel class of solvable nonlinear systems. Thus, it was shown in [29] that the class of Ermakov-Ray-Reid systems.

$$
\begin{aligned}
& \ddot{\alpha}=\frac{2}{\alpha^{3}} J(\beta / \alpha)+\frac{\beta}{\alpha^{4}} d J(\beta / \alpha) / d(\beta / \alpha), \\
& \ddot{\beta}=-\frac{1}{\alpha^{3}} d J(\beta / \alpha) / d(\beta / \alpha)
\end{aligned}
$$

parametrised in terms of arbitrary $J(\beta / \alpha)$, admits the Hamiltonian

$$
\mathscr{H}=\frac{1}{2}\left[\dot{\alpha}^{2}+\dot{\beta}^{2}\right]+\frac{1}{\alpha^{2}} J(\beta / \alpha)
$$

and Ermakov invariant

$$
\mathscr{E}=\frac{1}{2}(\alpha \dot{\beta}-\dot{\alpha} \beta)^{2}+\left(\frac{\alpha^{2}+\beta^{2}}{\alpha^{2}}\right) J(\beta / \alpha)
$$

which together allow the solution of the system (4.1) in an algorithmic manner. 
In the present nine-body context, the Ermakov connection shows that the system (3.3) may be embedded in the integrable triad of Hamiltonian Ermakov-Ray-Reid systems

$$
\begin{gathered}
x_{t^{*} t^{*}}^{*}=-\frac{2}{x^{* 3}} J_{1}\left(y^{*} / x^{*}\right)+\frac{y^{*}}{x^{* 4}} d J_{1}\left(y^{*} / x^{*}\right) / d\left(y^{*} / x^{*}\right), \\
y_{t^{*} t^{*}}^{*}=-\frac{1}{x^{* 3}} J_{1}\left(y^{*} / x^{*}\right), \\
z_{t^{*} t^{*}}^{*}=-\frac{2}{z^{* 3}} J_{2}\left(v^{*} / z^{*}\right)+\frac{v^{*}}{z^{* 4}} d J_{2}\left(v^{*} / z^{*}\right) / d\left(v^{*} / z^{*}\right), \\
v_{t^{*} t^{*}}^{*}=\frac{1}{z^{* 3}} J_{2}\left(v^{*} / z^{*}\right), \\
r_{t^{*} t^{*}}^{*}=-\frac{2}{r^{* 3}} J_{3}\left(r^{*} / s^{*}\right)+\frac{s^{*}}{r^{* 4}} d J_{3}\left(r^{*} / s^{*}\right) / d\left(r^{*} / s^{*}\right), \\
s_{t^{*} t^{*}}^{*}=\frac{1}{r^{* 3}} J_{3}\left(r^{*} / s^{*}\right),
\end{gathered}
$$

parametrised in terms of arbitrary $J_{1}\left(y^{*} / x^{*}\right), J_{2}\left(v^{*} / z^{*}\right)$ and $J_{3}\left(r^{*} / s^{*}\right)$. These systems augmented by the integrable triad (3.4) determine a solvable nine-component system which is reciprocally associated to a novel class of nine-body problems in which the original system (2.1)-(2.2) is embedded.

\section{References}

[1] J. Abdullaev, A.S. Desyatnikov and E.A. Ostravoskaya, Suppression of collapse for matter waves with orbital angular momentum, J. Optics 13 (2011) 064023.

[2] A. Bachkhaznadji and M. Lassaut, Solvable few-body quantum problems, Few Body Systems $\mathbf{5 6}$ (2015) $1-17$.

[3] A. Bachkhaznadji and M. Lassaut, Exactly solvable N-body quantum systems with $N=3^{k}(k \geqslant 2)$ in the $D=1$ dimensional space, Few-Body Systems 57 (2016) 773-791.

[4] F. Calogero, Solution of a three-body problem in one dimension, J. Math. Phys. 10 (1969) 2191-2196.

[5] F. Calogero, Solution of the one-dimensional N-body problems with quadratic and/or inversely quadratic pair potentials, J. Math. Phys. 12 (1971) 419-436.

[6] F. Calogero, Classical Many-Body Problems Amenable to Exact Treatments, Lecture Notes in Physics (Springer, Berlin, 2001).

[7] F. Calogero and C. Marchioro, Exact solution of one-dimensional three-body scattering problem with two-body and/or three-body inverse-square potentials, J. Math. Phys. 15 (1974) 1425-1430.

[8] F. Cornolti, M. Lucchesi and B. Zambon, Elliptic Gaussian beam self-focussing in nonlinear media, Opt. Commun. 75 (1990) 129-135.

[9] A.S. Desyatnikov, D. Buccoliero, M.R. Dennis and Y.S. Kivshar, Suppression of collapse for spiralling elliptic solitons, Phys. Rev. Lett. 104 (2010) 053902-1-053902-4.

[10] A. Donato, U. Ramgulam and C. Rogers, The 3+1-dimensional Monge-Ampère equation in discontinuity wave theory: application of a reciprocal transformation, Meccanica 27 (1992) 257-262.

[11] F.J. Dyson, Dynamics of a spinning gas cloud, J. Math. Mech. 18 (1968) 91-101.

[12] A.S. Fokas, C. Rogers and W.K. Schief, Evolution of methacrylate distribution during wood saturation. A nonlinear moving boundary problem, Appl. Math. Lett. 18 (2005) 321-328.

[13] A.M. Goncharenko, Y.A. Logvin, A.M. Samson, P.S. Shapovalov and S.I. Turovets, Ermakov Hamiltonian systems in nonlinear optics of elliptic Gaussian beams, Phys. Lett. A 160 (1991) 138-142.

[14] A.M. Goncharenko, Y.A. Logvin, A.M. Samson and P.S. Shapovalov, Rotating elliptic Gaussian beams in nonlinear media, Opt. Commun. 81 (1991) 225-230.

[15] A.M. Goncharenko, V.G. Kukushkin, Y.A. Logvin and A.M. Samson, Self-focussing of two orthogonally polarized light beams in a nonlinear medium, Opt. Quantum Electron 25 (1991) 97-104. 
[16] C.R. Guiliano, J.H. Marburger and A. Yariv, Enhancement of self-focussing threshold in sapphire with elliptical beams, Appl. Phys. Lett. 21 (1972) 58-60.

[17] A. Khare and R.K. Bhaduri, Some algebraically solvable three-body problems in one-dimension, $J$. Phys. A: Math. Gen. 27 (1994) 2213-2223.

[18] B. Konopelchenko and C. Rogers, Bäcklund and reciprocal transformations: gauge connections, in Nonlinear Equations in Applied Science, Eds. W.F. Ames \& C. Rogers (Academic Press, New York (1992) 317-362).

[19] D.C. Mattis, The Many-Body Problem: 70 Years of Exactly Solved Quantum Many-Body Problems (World Scientific, Singapore 1993).

[20] J. Moser, Three integrable Hamiltonian systems connected with isospectral deformations, Adv. Math. 16 (1975) 197-220.

[21] W. Oevel and C. Rogers, Gauge transformations and reciprocal links in 2+1-dimensions, Rev. Math. Phys. 5 (1993) 299-330.

[22] A.M. Perelomov, Integrable Systems of Classical Mechanics and Lie Algebras (Birkhausser, Basel 1990).

[23] J.R. Ray, Nonlinear superposition law for generalised Ermakov systems, Phys. Lett. A 78 (1980) 4-6.

[24] J.L. Reid and J.R. Ray, Ermakov systems, nonlinear superposition and solution of nonlinear equations of motion, J. Math. Phys. 21 (1980) 1583-1587.

[25] C. Rogers, Multi-component Ermakov and non-autonomous many-body system connections, Ricerche di Matematica to be published (2018).

[26] C. Rogers, C. Hoenselaers and J.R. Ray, On 2+1-dimensional Ermakov systems, J. Phys. A: Math. Gen. 26 (1993) 2625-2633.

[27] C. Rogers, B. Malomed, K. Chow and H. An, Ermakov-Ray-Reid systems in nonlinear optics, J. Phys. A: Math. Theor. 43 (2010) 455214.

[28] C. Rogers, B. Malomed and H. An, Ermakov-Ray-Reid reductions of variational approximations in nonlinear optics, Stud. Appl. Math. 129 (2012) 389-413.

[29] C. Rogers and H. An, Ermakov-Ray-Reid systems in 2+1-dimensional rotating shallow water theory, Stud. Appl. Math. 125 (2010) 275-299.

[30] C. Rogers and W.K. Schief, The pulsrodon in 2+1-dimensional magneto-gasdynamics. Hamiltonian structure and integrability, J. Math. Phys. 52 (2011) 083701.

[31] C. Rogers, Elliptic warm-core theory: the pulsrodon, Phys. Lett. A 138 (1989) 267-273.

[32] C. Rogers and W.K. Schief, On the integrability of a Hamiltonian reduction of a 2+1-dimensional nonisothermal rotating gas cloud system, Nonlinearity 24 (2011) 3165-3178.

[33] C. Rogers, Reciprocal relations in non-steady one-dimensional gasdynamics, Zeit. Angew. Math. Phys. 19 (1968) 58-63.

[34] C. Rogers, Invariant transformations in non-steady gasdynamics and magneto-gasdynamics, Zeit. Angew. Math. Phys. 20 (1969) 370-382.

[35] C. Rogers, Application of a reciprocal transformation to a two-phase Stefan problem, J. Phys. A: Math. Gen. 18 (1985) L105-L109.

[36] C. Rogers, On a class of moving boundary problems in nonlinear heat conduction. Application of a Bäcklund transformation, Int. J. Nonlinear Mechanics 21 (1986) 249-256.

[37] C. Rogers, On a class of reciprocal Stefan moving boundary problems, Zeit. Ang. Math. Phys. 66 (2015) 2069-2079.

[38] C. Rogers, Moving boundary problems for an extended Dym equation. Reciprocal connections, Meccanica 52 (2017) 3531-3540.

[39] C. Rogers, M.P. Stallybrass and D.L. Clements, On two phase filtration under gravity and with boundary infiltration. Application of a Bäcklund transformation, J. Nonlinear Analysis, Theory, Methods and Applications 7 (1983) 785-799.

[40] C. Rogers and T. Ruggeri, A reciprocal Bäcklund transformation: application to a nonlinear hyperbolic model in heat conduction, Lett. Il Nuovo Cimento 44 (1985) 289-296.

[41] C. Rogers and M.C. Nucci, On reciprocal Bäcklund transformations and the Korteweg-de Vries hierarchy, Physica Scripta 33 (1986) 289-292. 
[42] C. Rogers and S. Carillo, On reciprocal properties of the Caudrey-Dodd-Gibbon and KaupKuperschmidt hierarchies, Physica Scripta 36 (1987) 865-869.

[43] C. Rogers, The Harry Dym equation in 2+1-dimensions: a reciprocal link with the KadomtsevPetviashvili equation, Phys. Lett. 120A (1987) 15-18.

[44] C. Rogers and P. Wong, On reciprocal Bäcklund transformations of inverse scattering schemes, Physica Scripta 30 (1984) 10-14.

[45] C. Rogers and W.K. Schief, Bäcklund and Darboux Transformations. Geometry and Modern Applications in Soliton Theory (Cambridge Texts in Applied Mathematics, Cambridge University Press 2002).

[46] C. Rogers and W.K. Schief, Ermakov-type systems in nonlinear physics and continuum mechanics, in Nonlinear Systems and Their Remarkable Mathematical Structures N. Euler (ed) (CRC Press, Boca Raton Fl 2018).

[47] A. Rubino and P. Brandt, Warm-core eddies studied by laboratory experiments and numerical modelling, J. Phys. Oceanography 33 (2003) 431-435.

[48] B. Sutherland, Quantum many-body problems in one-dimension: ground state, J. Math. Phys. 12 (1971) 246-250.

[49] B. Sutherland, Exact results for a quantum many-body problem in one-dimension, Phys. Rev. A 4 (1971) 2019-2021.

[50] W.K. Schief, H. An and C. Rogers, Universal and integrable aspects of an elliptic vortex representation in 2+1-dimensional magnetogasdynamics, Stud. Appl. Math. 130 (2013) 49-79.

[51] W.G. Wagner, H.A. Haus and J.H. Marburger, Large scale self-trapping of optical beams in the paraxial ray approximation, Phys. Rev. 175 (1968) 256-266. 